\title{
Investigation of the law of distribution of cotton in the cleaning zone of the cleaner
}

\author{
Rasulov Ro'zimurod Xasanovich ${ }^{1}$, Prof. Mardonov Botir Mardonovich ${ }^{2}$, \\ Norbayeva Dilfuza Voxidovna ${ }^{3}$ \\ ${ }^{I}$ Candidate of Technical Sciences Associate Professor, Tashkent Institute of textile and light industry \\ ${ }^{2}$ Prof Tashkent Institute of textile and light industry \\ ${ }^{3}$ Doctoral student Tashkent Institute of textile and light industry
}

\begin{abstract}
The article investigates the regularity of the distribution of cotton in the cleaning zone of the cotton gin from the contaminants in the cleaning zone of the cotton pieces. It has been theoretically studied how the pressure, density, and velocities in a cotton raw material change after it has passed through each grate when it is subjected to an impact with the grate system.
\end{abstract}

Keywords: cleaner, saw drum, technological process, grate, dirt, speed, pressure, density, stationary motion, linear equation.

\section{INTRODUCTION}

In order to clean the raw cotton from large organic contaminants - husks, cotton stalks and leaves, in the process of processing raw cotton, cleaners are used, the main working organ of which is a saw drum. The base of the saw cleaner consists of a saw drum, a grinding brush, a grate grille and a brush separator drum.

Analysis of advanced technological processes of primary processing of cotton in the world, in particular, the leading countries in the field of cotton growing and processing, shows that in the technological process in Uzbekistan the cleaning process consists of a number of technological machines installed in a horizontal plane.

Operation of cleaning drums at speeds higher than the specified values is due to a sharp decrease in the quality of cleaning and, consequently, the formation of "fibrous waste" with an increase in seed damage.

Theoretical studies of the interaction of cotton pieces with the saw blade attached to the surface of the saw drum in the cleaning module of the cotton cleaner from large contaminants were conducted by E.F. Budin. The forces acting on the interaction of the cotton piece with the colossus were calculated, the models obtained during the shock process of cleaning were used to determine the technological and design parameters of the cleaner. However, all functional dependencies were constructed on the hypothesis of the impact of an absolutely rigid body, i.e., the solidarity-plasticity of the different degrees of solidarity and bond strength properties of the cotton pieces were not taken into account in the calculations.

In scientific research conducted by Y.S. Sosnovsky, the force interactions of cotton pieces with the grates were estimated, and a process model was proposed to determine the contact force of the cotton piece attached to the saw teeth. In calculating the process model, the author did not consider the strength parameters of the fiber bundle, so the calculated equations did not correspond to the experimental results.

The results of the study show that as the angular velocity of the saw drum increases, the force acting on the cotton mass and the probability of splitting the cotton pieces into smaller pieces increase.

In the works of A.Y. Lugachev, S.Fazilov, R.Z.Burnashev in the cleaning module the interaction of cotton pieces with a saw drum headset and a gluing brush is studied, and also developments are offered to increase cleaning efficiency in their use.

It was considered in the scientific researches carried out by the S.Fazilovs, in which impact parameters were checked and obtained experimentally in the interaction of cotton pieces with the surface of the grate. In this work, the development of a new, efficient grate construction was achieved.

The scientific research carried out by BN Yakubov on improving the cleaning process in order to reduce the emission of fibrous material in the cleaning module of the cotton from large contaminants arouse interest. Experimental dependencies were obtained by the author and a connection was established between the strength of the fiber in the bonding zone and its degree of adhesion with the sawing drum with the bonding brush. A theoretical model of the bonding process is also derived from the interaction of cotton pieces with the colossal surface. The calculations used the elongation dependence of the bond between the cotton pieces obtained experimentally. To reduce the leakage of fibrous material into the waste, it is proposed to install a connecting roller in the space between the grates, the last (from the sixth grate to the tenth) grates on the drum with an increasing groove along the parabola curve. But this proposal did not apply to the industry.

Theoretical and experimental studies of the process of interaction of cotton pieces with different types of grates are conducted by S. Fazilov. The author obtained a number of models of the cleaning process that characterize the shape of the grate, resulting in a variable curve (according to the Archimedes spiral) grate with an alternative shape for each type of cotton during the cleaning process. However, in the analysis of the interaction of cotton pieces with the surface of the grate, the properties of the fibrous material and the uniformity characteristics of the impurity in relation 
to the fiber were not taken into account.

The scientific work carried out by K.T. Olimov considered the tasks of alternating the acceleration of the process of interaction of spring-loaded grates with cotton pieces in the cleaning module of the cotton from large contaminants. However, these works were not put into production due to the complexity of the construction of the drive.

M.Agzamov studied the process of accelerating the separation of pollutants in rotating grates and achieved positive results. However, due to the complexities in dynamic modes and driving kinematics, it was not possible to apply this proposition to production.

M.J. Koshakova considered the implementation of the cleaning process using vibration. This process has many advantages as it retains the quality characteristics of the fiber compared to the traditional process, but due to the low cleaning efficiency and work efficiency, this construction has not been used in industry.

D.R. Khaitov conducted research on the elemental grates twisted on supports. In this case, the clogging of the cotton between the saw drum and the grate "softens" and dampens the impact process when a piece of cotton hits the grate. However, in the working zone of the ginner there is a rapid process of interaction of the pieces of cotton wool, which reduces the efficiency of the proposed device, taking into account the increased impact of elastic-deformation of cotton.

When the flow of raw cotton is under the impact of a system of grate, we theoretically study the changes in pressure, density, and velocities in it after passing through each grate. To model this process, we assume the following assumptions.

Assuming that the mass of the cotton is stationary and the motion of the stream is stationary, then the productivity in the stream is constant in the zone where the grates are located, and is equal to Q0 contaminants separate from the stream do not affect productivity Q0.

The motion of the flow is assumed as one passenger between the grates.

The radius of the grates increases along the arc where they are located, keeping the distance between them.

An arbitrary grate is in contact with a cotton stream (environment), and the immersion of the grate in the medium is determined according to Hertz's law, or practice. We denote the velocity, pressure, and density (parameters) of the flow between each grate and the section surface by vi, pi, and Si. (i=1.n). n- the number of grates.

We determine the pressure parameters between the first and second grates.

Suppose the initial parameters of the current (except for the grates zone) are $\rho_{9}, v_{0} h_{0}$ and $S_{0}$. Let the flow thickness $h_{0}$ before exposure to the first coil be equal to the working efficiency of the flow $Q_{9}=\rho_{9} v_{0} h_{9} L$, where $\mathrm{L}$ is the length of the drum.

The zone of interaction with the first grate is ABSD, in which we determine the flow parameters. We direct Ox axis along the DS section. We place the coordinate head at point $\mathrm{O}$ (Figure 1).

An arbitrary mn surface (perpendicular to the plane) is defined by the following formula:

$$
S=\left(h_{0}-u_{0}+\frac{x^{2}}{2 R_{0}}\right) L \quad-x_{0}<x<x_{0}
$$

here h0 initial thickness of the raw material, $x_{0}=\sqrt{2 u_{0} R_{0}} u_{0}$ - the maximum immersion of the grate in the flow, its value is determined experimentally, according to Hertz's law, or $R_{0}$-radius of the grate, L- the length of the drum shaft. We construct the Euler equation under the condition of stationary motion for the separated element:

$$
-[S p+d(S p)]+S p-q L d x=\rho v S d v
$$

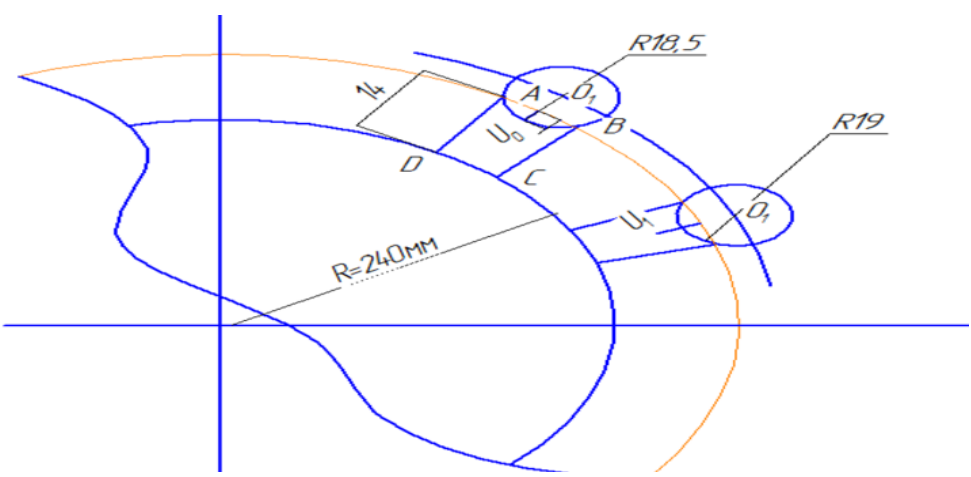

Figure 1. View of the cleaner cotton cleaning section from large contaminants.

here $q=f p$ - side pressure, $k$ - pressure coefficient, $f=f_{1}+f_{2}, f_{1}, f_{2}$ - accordingly coefficients of friction between the cotton and the drum and the grate.

Taking note the equation $S=b(x) L$ and the expression $q$, the expression is $\mathrm{dx}$, we obtain the following 
equation.

$$
\begin{aligned}
& \rho v b \frac{d v}{d x}=-\frac{d(p b)}{d x}-k f p \\
& \qquad b=\left(h_{0}-u_{0}+\frac{x^{2}}{2 R_{0}}\right) \\
& \text { Here: }
\end{aligned}
$$

Equation (3) contains unknowns r, v, p, we use two conditions to complete it.

First, the flow is a stationary condition

$$
\rho v b=\rho_{0} v_{9} b_{0}=Q_{0} / L
$$

The second condition is that the equation of state of the environment must be reasonable.

To do this, we take the relationship between pressure and density, at small values of pressure ( $\rho v b=\rho_{0} v_{9} b_{0}=Q_{0} / L$ ) the linear relationship between them is appropriate.

$$
\rho v b=\rho_{0} v_{9} b_{0}=Q_{0} / L
$$

$p_{0}$ initial pressure in the raw material, $B$-experience coefficient.

Using connections (4) and (5), we determine the expression for pressure by velocity

$$
\begin{gathered}
p=p_{0}+\frac{1}{B}\left(\frac{v_{0} h_{0}}{v b}-1\right) \\
\left(1-\frac{c^{2}}{v^{2}}\right) \frac{d v}{d x}=-\frac{c^{2}}{v_{0} b_{0}}\left[b^{\prime}+f k\left(p_{0} B-1\right)\right]-\frac{c^{2} f k}{v b}
\end{gathered}
$$

$b^{\prime}=x / R_{0}$ putting the expression into this equation:

$$
\frac{d v}{d x}=-\frac{c^{2}}{v_{0} b_{0} a}\left[\frac{x}{R_{0}}+f k\left(p_{0} B-1\right)\right]-\frac{c^{2} f k}{v b a}
$$

Here $a=1-c^{2} / v^{2}, c=\sqrt{K / \rho_{0}}, K=1 / B$ is the modulus of compression of the ambient volume Equation (7) determines the velocity of the current in the interval in contact with the column. The equation is integrated in the following $x=-x_{0}$ да $v=v_{0}=Q_{0} / \rho_{0} h_{0} L$ initial condition.

When we change expression (8) to (9), we estimate the ratio error as $\Delta p=p-p_{0}$ a percentage at different values of the coefficient. We determine the ratio $\delta_{1}$ of their seperation $\delta_{1}$.

$$
\delta=\frac{100\left(\delta_{1}-\delta_{2}\right)}{\delta_{1}}=100 B^{2} \Delta p^{2}
$$

The following table shows the maximum values pm for each error $\delta(\%)$ at different values $\Delta p_{m}$ of the given coefficient $B$ of the pressure $\Delta p=p_{0}-p$. If the error $\delta(\%)$ is given in the formula (9) used to solve the problem, then the $\Delta p \leq \Delta p_{m}$ condition for pressure must be satisfied during the calculation. For example, if the raw material is known $B=0.0015 \Pi \mathrm{Ia}^{-1}$, the calculated pressure should not exceed $\Delta p=p_{0}-p \quad 115.5 \mathrm{~Pa}$ based on the results in the table so that the error in using formula (9) does not exceed 3\%, the pressure $\Delta p=p_{0}-p$ 210. 8Pa should not exceed $8 \mathrm{~Pa}$.

Table.

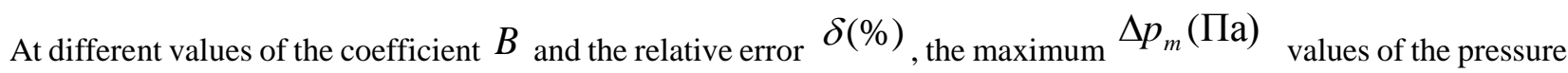
$\Delta p$ pressure using the formula (9)

Maximum values of pressure at different values of the coefficient and relative error when using formula (9)

\begin{tabular}{|l|l|l|l|l|l|l|l|l|l|l|}
\hline & \multicolumn{4}{|l|}{$B=0.0005 \Pi \mathrm{Ia}^{-1}$} & \multicolumn{5}{l|}{$B=0.001 \Pi^{-1}$} \\
\hline$\delta(\%)$ & 1 & 3 & 5 & 8 & 10 & 1 & 3 & 5 & 8 & 10 \\
\hline
\end{tabular}




\begin{tabular}{|l|l|l|l|l|l|l|l|l|l|l|}
\hline$\Delta p_{m}(\Pi \mathrm{a})$ & 200 & 346.2 & 447.2 & 565.7 & 632.4 & 100 & 173.2 & 223.6 & 282.8 & 316.2 \\
\hline \multicolumn{8}{|c|}{} & \multicolumn{10}{|c|}{$B=0.002 \Pi^{-1}$} \\
\hline$\delta(\%)$ & 1 & 3 & 5 & 8 & 10 & 1 & 3 & 5 & 8 & 10 \\
\hline$\Delta p_{m}(\Pi \mathrm{a})$ & 67.7 & 115.5 & 149.1 & 188.6 & 210.8 & 50 & 86.6 & 111.8 & 141.4 & 158.1 \\
\hline
\end{tabular}

Using formula (9), we make the equation look like a linear equation:

$$
\left(M^{2} h_{0}-b\right) \frac{d v}{d x}=-\left(b^{\prime}+f k\right)\left(\left[p_{0} B+1\right) v_{0}-v\right]
$$

If we put the expression of $b$, the variables of the equation are separated

$$
\frac{d v}{\left[\left(p_{0} B+1\right) v_{0}-v\right]}=\frac{2\left(x+R_{0} f k\right)}{a^{2}+x^{2}} d x \text { if }-x_{0}<x<x_{0}
$$

Here, the speed of wave propagation in the medium. Since the raw cotton is a deformable bonding medium, one of the indicators of such an environment is the vibrations that propagate in it, they have a specific frequency (wavelength) and its value is equal $\omega=c / l$. If $B=0.001 \Pi \mathrm{a}^{-1}, \rho_{0}=40 \kappa 2 / \mathrm{M}^{3}$, then it equals to $c=5 \mathrm{M} / \mathrm{c}$.If the efficiency of the cleaning machine is equal to $Q=5000 \mathrm{~kg} / \mathrm{hour}, Q=7000 \mathrm{~kg} / \mathrm{hour}, Q=9000 \mathrm{~kg} / \mathrm{hour}$ at $h_{0}=0.014 \mathrm{M}, L=1.9 \mathrm{M}$ , $\rho_{0}=40$ values the velocity of the raw material in the cleaning zone on the basis of $\mathrm{v}$ is $1.31 \mathrm{M} / \mathrm{c}, 1.83 \mathrm{M} / \mathrm{c}$, 2.35M.c , $2.87 \mathrm{M} / \mathrm{c}$ respectively, is equal to the inequality $M<1$.

\section{REFERENCES:}

1. Zikriyoev E.Z. Initial processing of cotton. // Tashkent, "Labor", 2002.

2. Cotton: World Statistics. http://www.ICAC.org; https: //www/statistica.com.

3. F.B.Omonov. "Reference on the initial processing of cotton". Tashkent, Voris Publishing House, page 400. 2008.

4. Rosulov Rk1 * and Saphoyev AA. To the Problems of Cleaning of Hard -grades Raw Cotton. Journal of Textile \& Engineering. 2015, 5-2.

5. Budin E.F. Investigation of grate-serrated working bodies of raw cotton purifiers for machine harvesting of medium-fiber varieties: Diss.... Cand. those. sciences. Tashkent: TITLP, 1968 -- 156 p.

6. Sosnovskiy Y.S. Research and selection of parameters of cleaners of fine-staple raw cotton machine collection: Diss ... those. sciences. -Tashkent. TITLP, 1971 - 222 p.

7. A.E. Lugachev, S. Fazilov, R.Z. Burnashev. On fixing raw cotton on the serrated surface of the cleaner Coll. Cotton industry, Tashkent, 1978 - No 6, pp. 16-18.

8. R.Z.Burnashev, A.E. Lugachev, S. Fazilov. Experimental study of the impact interaction of raw cotton volatiles with a grate of a coarse litter cleaner. Cotton industry -Tashkent, 1980- No 1, S. 7-8.

9. B.N. Yakubov Improving the process of cleaning raw cotton from weeds in saw-type cleaners in order to reduce the loss of cotton fiber mass: Diss.... Cand. those sciences - Tashkent: TITLP, 1985 -- $156 \mathrm{p}$.

10. S. Fazilov K. T. Olimov Grizzly units of raw cotton cleaners from large trash impurities with adjustable parameters: Diss .... cand. those sciences. - Tashkent, 1985 - 142 p.

11. K.T. Olimov. Development and substantiation of the parameters of grates on elastic supports for cleaners of raw cotton from large weeds: Diss.... Cand. those. sciences - Tashkent: TITLP, 1998 -- 135 p.

12. M.Agzamov. Increasing the efficiency of cleaning raw cotton by machine picking in the barbed sections of the cleaners: Diss .... cand. those. sciences - Tashkent: TITLP, 1985 -- 196 p.

13. M.J. Koshakova. Cleaning cotton using vibration: Diss .... cand. those. sciences - Tashkent: TITLP, 1985 -$236 \mathrm{p}$.

14. D.R. Xaitov. Increasing the efficiency of cleaning raw cotton by improving the working bodies of cleaners of large litter: Diss .... cand. those sciences - Tashkent: TITLP, 1990 -- 212 p. 